\title{
A diabetic hypertension treatment program for the underserved in rural Dominican Republic
}

\author{
Miguel Cruz, Linda Filipi, Josefina Estevez, Evaristo Marte, Han Dethlefs, Rachel Dowd, \\ Andras Legner, Vinayak Kottoor, Charles Filipi* \\ Department of Surgery, Creighton University Medical Center, Omaha, USA \\ Email: ${ }^{*}$ cjfilipi@creighton.edu
}

Received 9 February 2012; revised 23 March 2012; accepted 10 April 2012

\begin{abstract}
Background: Worldwide, diabetes and hypertension are leading causes of preventable end-organ disease. The prevalence of these diseases in the Dominican Republic is high and the end stage complications common. A treatment program utilizing modern protocols often thought too complex for the rural poor was initiated. Methods: With local government permission, a pilot study utilized Dominican physicians and local healthcare advocates (cooperadores) to obtain medical histories and physical exams as well as to determine healthcare needs specifically for type 2 diabetes and hypertension. A glycosylated hemoglobin (A1c) was used to identify and stratify diabetes patients. Blood pressure parameters per established standards were used to identify patients with hypertension. As indicated, pharmacotherapy was initiated (see treatment protocols), multiple forms of education and awareness building utilized, and a protocoldriven follow-up program maintained under weekly review. Results: In fifteen months, 1405 patients were screened, 229 type 2 diabetes patients, 59 pre-diabetes patients, and 98 hypertension patients were identified and enrolled for education, treatment, and follow-up. Normalization of blood pressure in hypertensives at 6 month follow-up was $78 \%$ and reduction of A1c values to $<7.0$ was $79.8 \%$. Conclusion: Utilizing a best practice approach per internationally agreed-upon parameters has been shown here to be not only relevant but capable of improving outcomes in a developing world setting. A model incorporating standard of care, education, and integration of local resources as established suggests that further study is warranted to evaluate the long-term benefits as well as secondary outcomes of this approach to local populations.
\end{abstract}

Keywords: Diabetes; Pre-Diabetes; Hypertension;

*Corresponding author.
Treatment; Developing; Country

\section{INTRODUCTION}

Type II Diabetes has become a global epidemic. According to the International Diabetes Federation 285 million people worldwide suffer from diabetes and 344 million have impaired glucose tolerance. Unfortunately, these statistics are expected to increase over time. By 2030 the number of people living with diabetes is projected to rise to 438 million and impaired glucose tolerance to 472 million. The World Health Organization [1] states that $80 \%$ of the world's diabetes occurs in low to middle income countries. The prevalence in the Dominican Republic is $11.2 \%$ and is the third main cause of death behind heart attacks and traffic accidents [2].

Hypertension is one of the largest contributors to cardiovascular disease (CVD) which is the number one cause of death globally. It is estimated that 17.1 million people died in 2004 from CVD, which represents $29 \%$ of all deaths in the world, 5.2 million from stroke and 7.2 million as a result of coronary heart disease [3]. Acosta et al. [4] completed a 10/66 cross-sectional based population survey in the Dominican Republic. Of 2011 people 65 years and older, $73 \%$ of the group were hypertensive, $17.5 \%$ were diabetic, and $39.6 \%$ suffered from metabolic syndrome (hypertension, insulin resistance, dyslipidemia, and obesity).

We conducted a field trial working with the Institute for Latin American Concern (ILAC), located in Santiago, Dominican Republic. ILAC is a non-profit organization that provides education and health care for rural Dominicans. The population ILAC serves has little access to health care. A pool of 160 cooperadores de salud (health promoters), who are elected by their respective villages, receive bi-annual educational updates and are supervised by two full time ILAC physicians. The cooperadores de salud provide education and assistance on basic health issues for their village members. The program has existed for over 25 years and is highly respected throughout 
the country.

The cooperadores de salud along with the ILAC administrative staff organized a needs assessment, in which 3 U.S. based physicians and 2 U.S. diabetic educators saw 390 diabetic patients over 2 days. A1c values, blood sugars, urine microalbumin, blood pressures, serum creatinines, eye and foot examinations were conducted on all patients. Five patients had a blood sugar over 500 $\mathrm{mg} / \mathrm{dl}, 16$ had significantly elevated creatinines and were referred to a nephrologist, 120 had abnormal eye examinations and were referred for retinal or cataract surgery evaluation, 6 patients had unhealed foot or leg ulcers, and more than $50 \%$ of the patients required modifications of their diabetic medications. One juvenile diabetic was severely malnourished and had no insulin. There was an obvious need for improved care and because the ILAC catchment area has at least 150,000 citizens (54\% rural), it was apparent there were many untreated diabetics. In addition, Dominican specialists [5] informed the team that over $50 \%$ of emergency room visits in Santiago are related to end stage renal, heart, eye, neurologic and extremity complications secondary to diabetes mellitus and hypertension.

A Dominican Republic report in 2008 [6] showed the level of poverty to be higher in rural areas with extreme poverty being present in $12.5 \%$ of rural homes. The degree of social inequality present in the province of Sanchez Ramirez is one of the highest in the country, with $53.4 \%$ of the homes being considered poor. The 2002 unemployment rate was $20.7 \%$ whereas the national unemployment rate was $17.8 \%$ and the high school graduation rate in Sanchez Ramirez was 18.2\%. Fifteen percent of the population did not have any primary education at the time of the survey.

\section{METHODS}

The Institute of Latin American Concern (ILAC) board gave permission for the initiation of the Sanchez Ramirez program and funding was obtained from the Dominican Development Alliance, a division of USAID and Chicago Cubs Charities. The primary goal of the Diabetes Hypertension program founders was to reduce the complications of these diseases in the long term. Initial outcome measure goals included reducing A1c levels to $<7.0$ in $35 \%$ of diabetic patients at 6 month follow-up and normalizing blood pressure in $25 \%$ of the hypertensive patients. We chose an ILAC clinic in the rural town of Comedero Abajo in the province of Sanchez Ramirez to initiate screening and treatment. The clinic serves the town and its surrounding mountain villages. The main barriers to health care in the area include low health literacy, a lack of transportation to urban health care facilities, and cultural myths such as diabetes is due to fate or trauma, and that insulin causes blindness.
Permission to work in Sanchez Ramirez was obtained from the minister of health. A Dominican medical director, a physician living in Comedero, and a nurse living near Comedero were hired and 5 cooperadores de salud in the region agreed to assist with screening, education, and treatment. A small stipend was provided for the cooperadores de salud. The region for treatment and screening is shown in Figure 1. A protocol was written and treatment algorithms based on American Diabetes Association and American Heart Association standards were agreed upon (Figures 2 and 3) and implemented. A1c correction was defined as a level $<7.0 \%$ and blood pressure normalization was three consecutive blood pressures $<130 / 80 \mathrm{mmHg}$ (diabetic patients) or $<140 / 90$ mmHg for non-diabetic patients. Only patients that could not afford treatment were screened and enrolled. Local government funded clinics provide care but many diagnosed with diabetes or hypertension cannot afford the medications and a comparable health provider network is not provided. Fifty patients with a previously established diagnosis and on medication, were enrolled in the ILAC program because they did not have access to continued care or could not afford treatment costs.

Screening was first performed by administering a questionnaire $(n=669)$ that determined risk factors and if at risk the patients then had a blood sugar and blood pressure assessment. An A1c of $>5.8 \%$ and $<6.4 \%$ was used for a diagnosis of pre-diabetes and $>6.5 \%$ for diabetes. If the blood pressure exceeded 140/90 $\mathrm{mmHg}$ in non-diabetics it was repeated and if still elevated the patient was treated. Because both conditions were found to be prevalent, we changed screening to simply obtaining a blood sugar and a blood pressure. If either was elevated on two occasions they were enrolled in their respective program and if both parameters were elevated, the patient was treated for both conditions. A history and physical examination was conducted by a program physician and initial blood work was drawn including an A1c, liver function tests, and a creatinine. Initial laboratory blood work performed locally was found unreliable and thereafter was performed in a certified laboratory in Santa Domingo. Subsequently a new Point of Care A1c machine (Siemens, Inc.) was donated and cartridges were purchased.

Medications (Teva Pharmaceuticals), creatinine cartridges, glucometers (Abbott Laboratories) and an ISTAT (Creighton University Medical Center) were donated. Lancets and strips were purchased. Initially blood sugars were obtained only at the time of clinic visits, although some patients, with the help of the cooperadores, were able to use glucometers at home.

We used Metformin ${ }^{\text {TM }}$ because of its first-line status and safety profile, and Glipizide ${ }^{\mathrm{TM}}$ was added for inadequate response to Metformin $^{\text {TM }}$. Patients that could not 

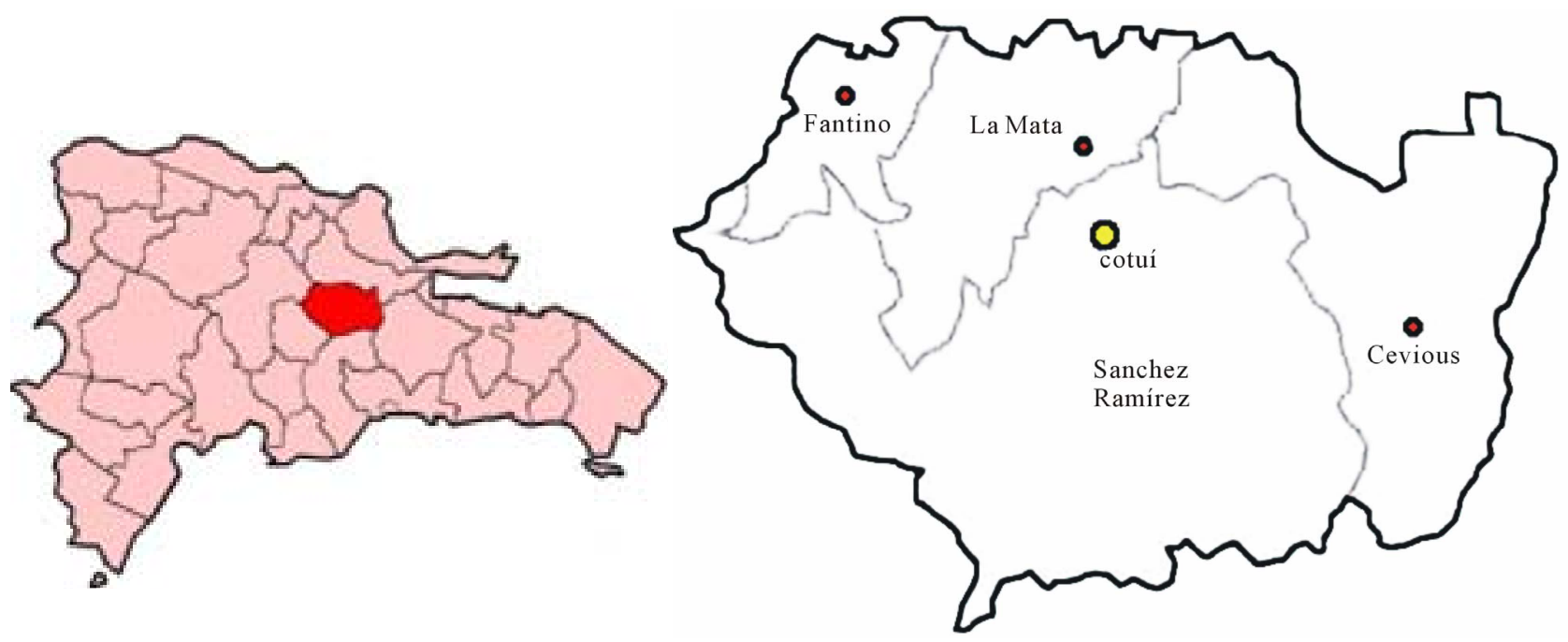

Figure 1. The Sanchez Ramirez province in north central Dominican Republic.

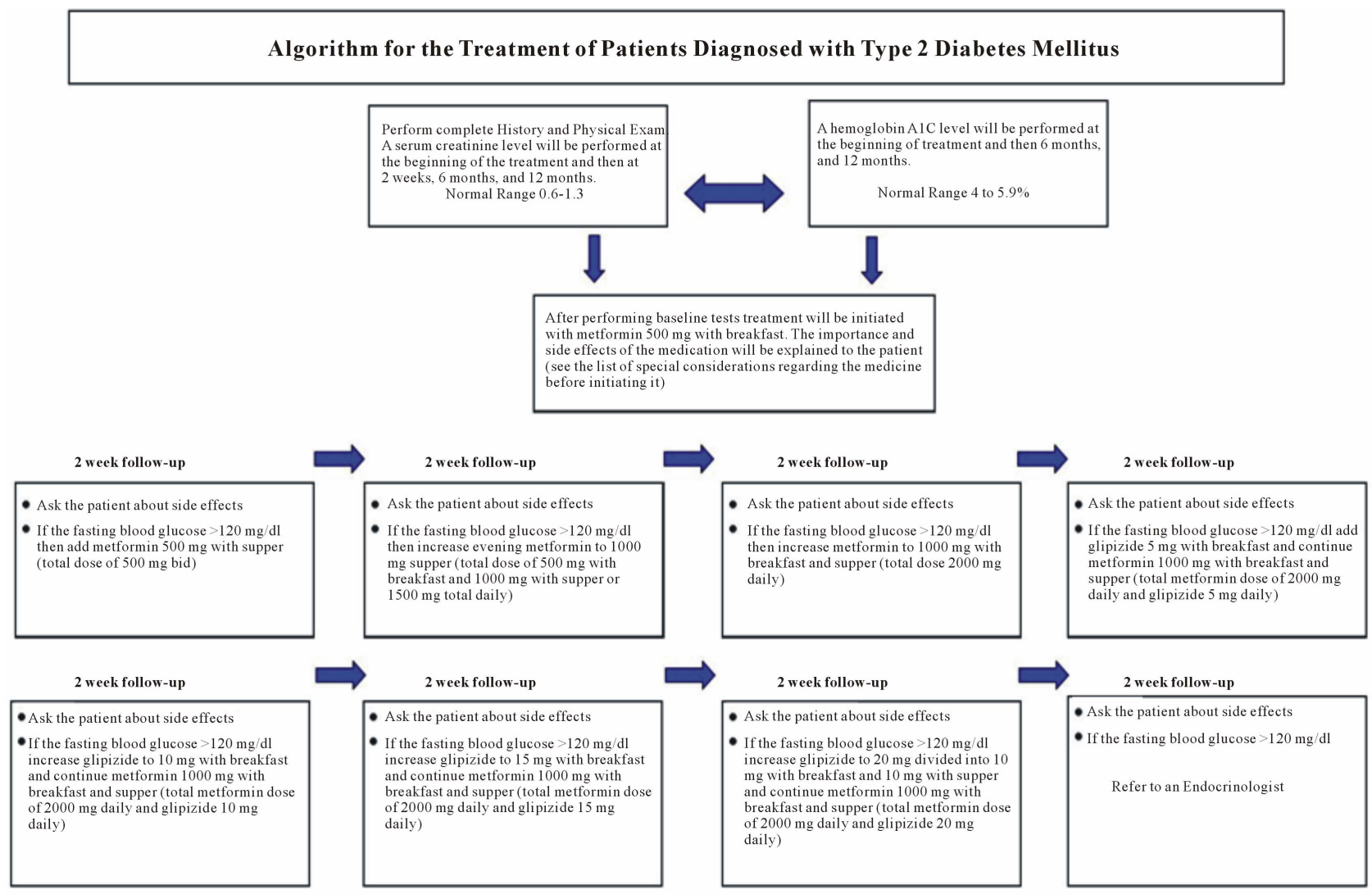

Special Considerations

- Metformin should not be used in patients with signs or symptoms of congestive heart failure or liver failure.
- Metformin is contraindicated in patients with renal insufficiency as evidenced by a serum creatinine $>1.5 \mathrm{mg} / \mathrm{dl}$ in me or $>1.4 \mathrm{mg} / \mathrm{dl}$ in women
- Metformin should be avoided in patients $\geq 80$ years of age with a glomerular filtration rate $<60 \mathrm{ml} / \mathrm{min}$ or elevated serum creatinine.
- All patients using metformin should be advised to suspend the medicine 48 hours prior to and 48 hours after any radiology studies in which
iodinated contrast is used.
- Patients using glipizide should be educated on the risk and symptoms of hypoglycemia, and that they should ingest a source of glucose if such
symptoms should rise. These patients should receive a monitor to be able to check their BS at home.

Figure 2. Algorithm for the evaluation and treatment of patients with type 2 Diabetes Mellitus. 


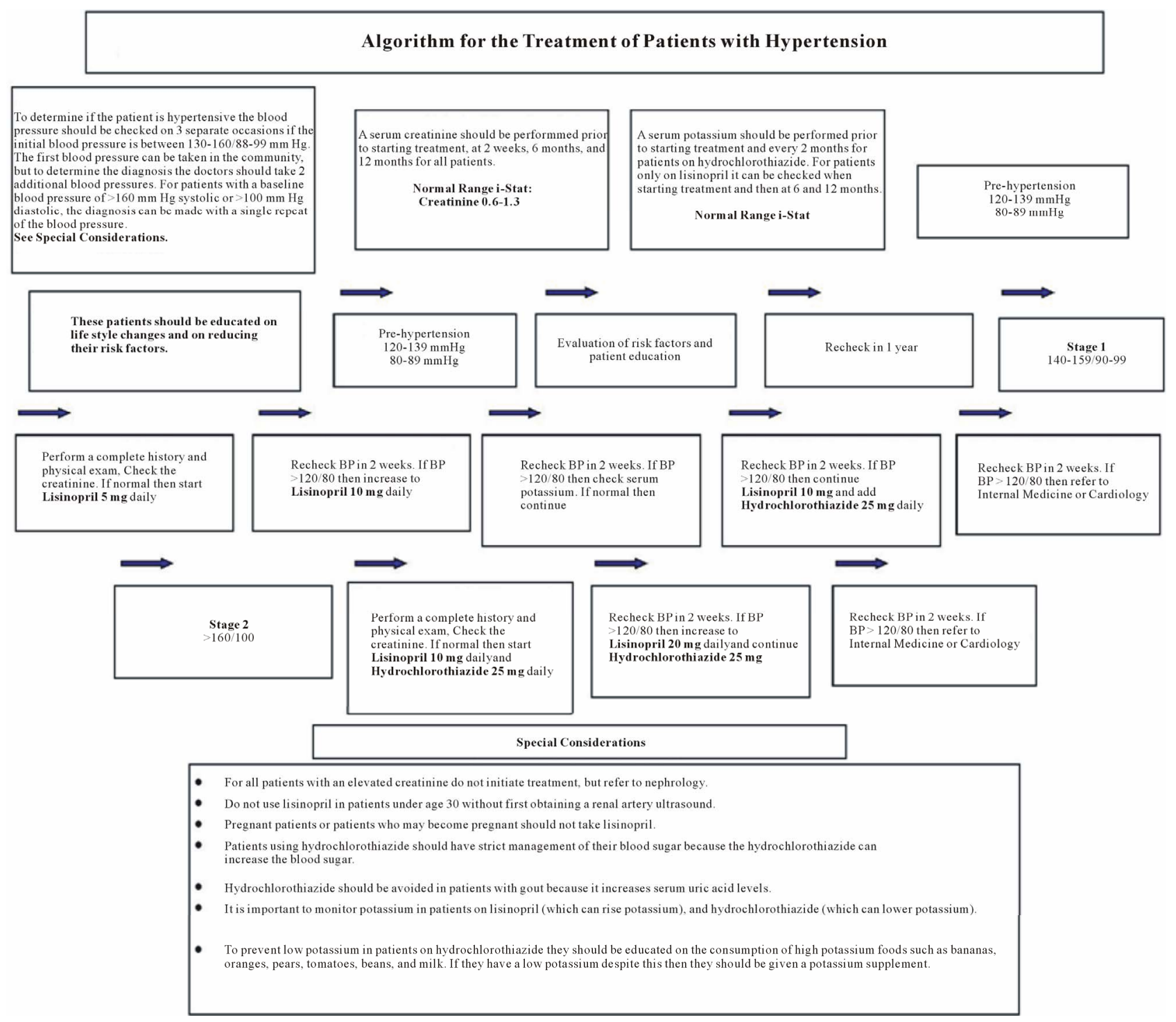

Figure 3. Algorithm for the evaluation and treatment of patients with hypertension.

be controlled with oral anti-hyperglycemics were referred to an endocrinologists for insulin therapy $(n=5)$ and 3 patients with elevated serum creatinines were referred to a nephrologist. Two patients had elevated liver function tests and were referred to a gastroenterologist. Although referred to outside specialists, their diabetic education was continued. Hypertension was treated with titrated doses of Lisinopril and Hydrochlorathiazide was added when needed. For hypertension patients a simple education pamphlet was given which explained the diagnosis, and the importance of follow-up visits and medication adherence.

The cooperadores de salud first had a four hour inservice on the basics of diabetes self management by two U.S. certified diabetes educators. To provide a strong educational foundation for the cooperadores de salud and patients we utilized a CD series called Rosa's Story. De- veloped by Enrique Caballero, M.D. [6], the set of CD’s teaches diabetes self management through story telling. The story is not only appropriate for those with lower levels of education but is culturally sensitive.

After patient diagnosis, cooperadores de salud then started the 5 session education series with newly identified pre-diabetic and diabetic patients. Small patient groups (8 - 12 people) were formed, and one or more of the patient's family members attended the sessions. The CD was played for the group and the cooperadores de salud answered related questions. Sessions were repeated when necessary. The cooperadores de salud continued to contact the patients approximately once a week to remind them about medications, appointments and to answer questions.

Two U.S. based medical consultants and two diabetic nurse educators designed the treatment algorithms and a 
spread sheet for tracking patient data. Weekly phone conferences were conducted with the Dominican Medical Director, Dr. Cruz. Compliance with enrollment goals and other logistic matters including medications, diabetic equipment and inventory were monitored by Dr. Filipi. Physician algorithm compliance was monitored monthly by Dr. Cruz and periodically by the U.S. based doctors and nurses during in person visits. A computer notebook was given to the local Dominican physician, Dr. Josefina Estevez, and she recorded data on the spreadsheet.

\section{RESULTS}

After 15 months a total of 1,405 patients were screened and as a result 229 diabetic patients, 59 pre-diabetic patients and 98 hypertension patients had treatment initiated. Education, life style changes, cooperadores de salud follow-up, Metformin ${ }^{\mathrm{TM}}$ once or twice a day, and when necessary in combination with a sulfonylurea (Glipizide $^{\mathrm{TM}}$ ) was used when indicated by protocol. In several cases Glipizide ${ }^{\mathrm{TM}}$ was used alone. Patients on Glipizide only, were elderly patients with co-morbidities contraindicating the use of Metformin or those that had come into the program on Glipizide ${ }^{\mathrm{TM}}$ with good control.

Two hundred and thirty-seven diabetic and pre-diabetic patients have been educated using Rosas' Story. A total of 194 patient sessions have been conducted. One hundred and fourteen patients have completed all 5 sessions. We estimate that 400 additional people have attended the sessions. A total of 21 patients have been lost to follow-up and 100 patients have returned for 9 month follow-up.

Hypertension, pre-diabetes, and diabetes patients follow up visit frequency is shown in Figures 4-6. At 9 months 68 hypertension patients had 1.5 average followup visits, 88 pre-diabetic patients 1.6 average follow-up visits, and 196 diabetic patients had 2.2 visits on average. Medication usage for the different diagnoses is shown in Figures 7 and 8.

Eighty-seven of 109 diabetic patients at the 6 month follow up had an A1c $<7$ (79.8\%). The blood pressure normalization rate was $78 \%$ in hypertension patients at the 6 month follow up. There were no episodes of hypoglycemia, hyperosmolar state, or ketoacidosis reported by patients on treatment during the study period. Eleven patients had a blood sugar over $400 \mathrm{mg} / \mathrm{dl}$ at screening. Four had been on insulin previously but were off medication and 5 had been on oral medication and were also off their medicines. Two had not been previously diagnosed.

\section{DISCUSSION}

Two hundred and sixty two patients have been treated for the first time for diabetes and hypertension. Follow-up

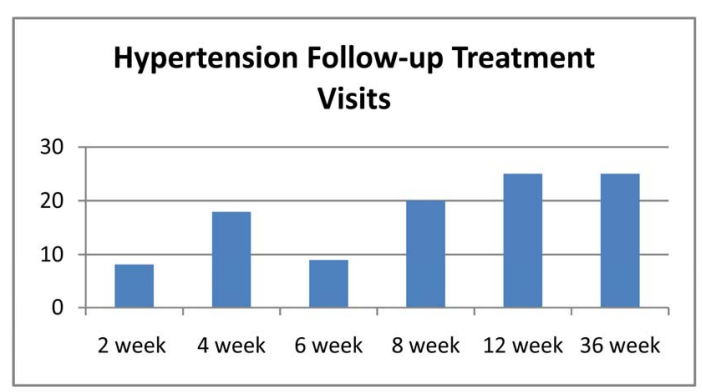

Figure 4. Follow-up visits for Hypertension patients.

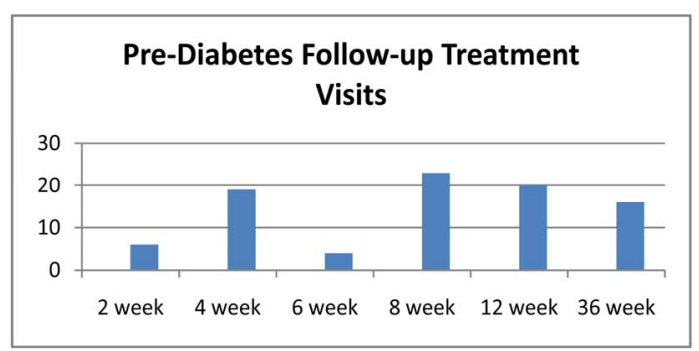

Figure 5. Follow-up visits for pre-diabetes patients.

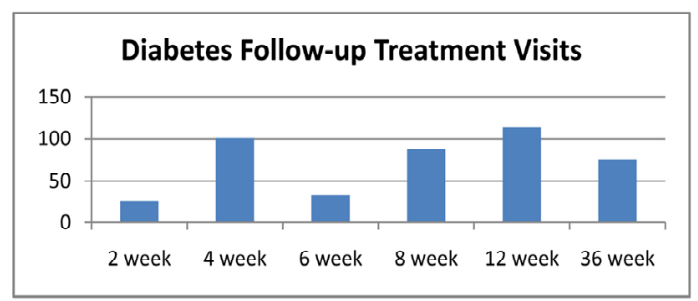

Figure 6. Follow-up visits for diabetes patients.

has ranged from 3 - 15 months and baseline and follow-up A1c and blood pressures were obtained. The initial results exceeded our expected outcomes and are attributed to physician algorithm compliance, patient medication adherence and less advanced disease. Patients requiring insulin were referred to an endocrinologist. Medication adherence was due to the CD education group program "Rosas' Story” [7], clinic visits, and frequent follow-up cooperadores de salud interactions with the patients.

These results demonstrate the effects of implementing a standardized approach to type 2 diabetes and hypertension using algorithms based upon consensus guidelines. As discussed above, this local experience is reflective of the challenges of diabetes and hypertension management worldwide. Practitioners, policy makers, and patients alike will attest to the significant hurdles and ongoing difficulties in managing these chronic diseases. For populations in the developing world for whom morbidity and mortality are shifting from acute illnesses such as infections, to chronic illnesses, the need to find ways in which to facilitate such care is self-evident. JR Guzman 


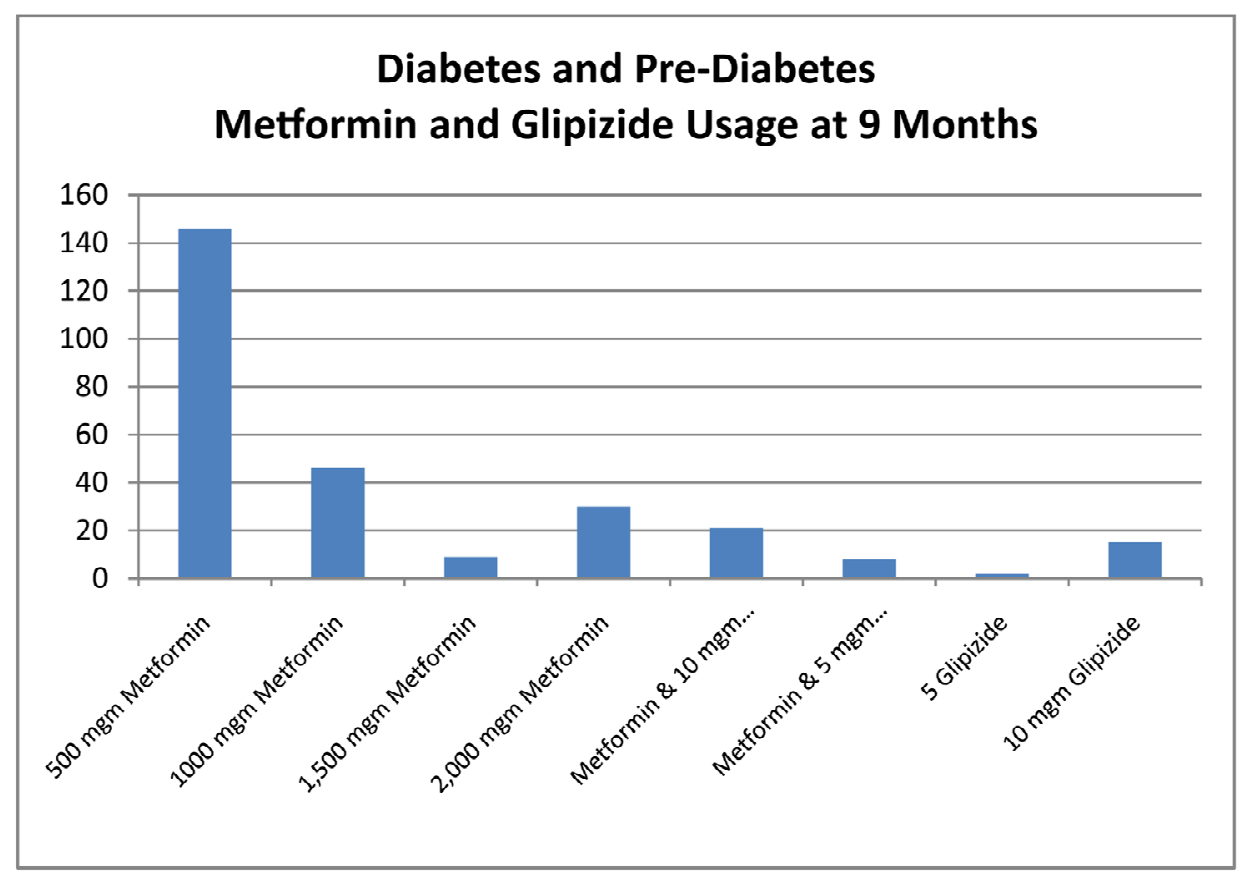

Figure 7. Medication usage for pre-diabetes and diabetes patients at 9 months.

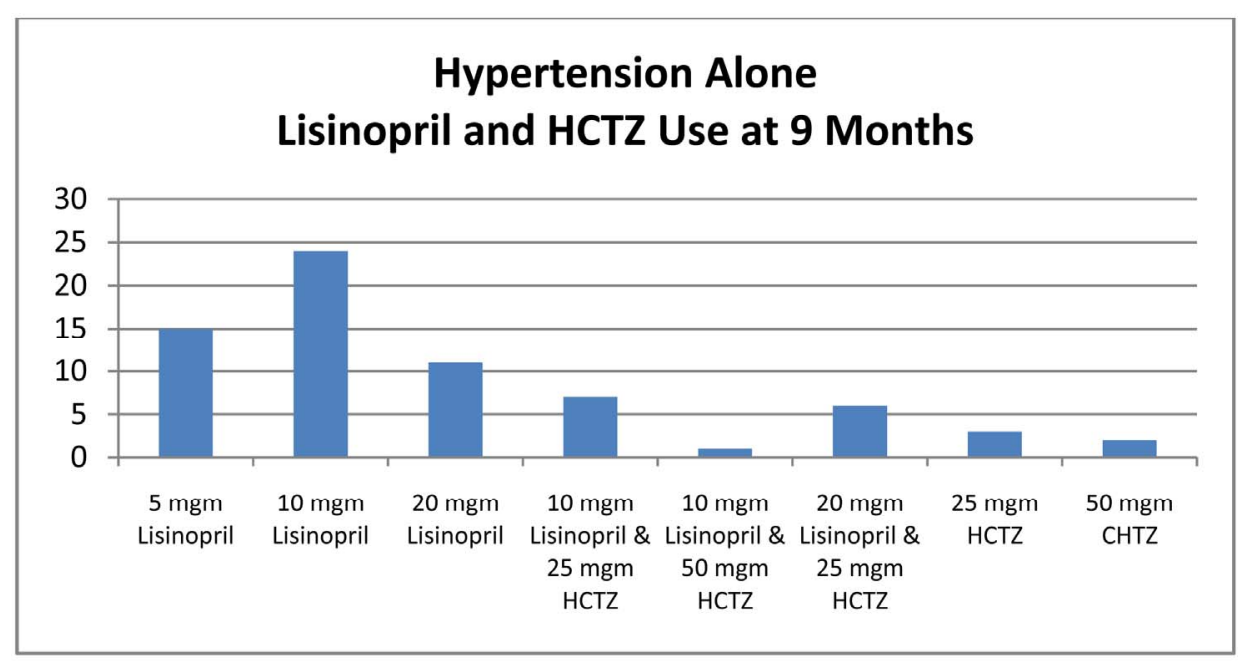

Figure 8. Medication usage for hypertension patients at 9 months.

et al. [8] proposed a consensus statement for the treatment of type 2 diabetes in Latin America. Not surprisingly, the underlying management guidelines are essentially unchanged from consensus statements around the globe. The most notable points which are of particular relevance to this project include finding ways to bring the benefits of a consensus approach to patients in the Dominican Republic utilizing local resources including local providers. Overcoming the lack of availability of essential tests such as the A1c, liver, and kidney function tests was a barrier that needed to be resolved. The shortage of general practitioners to administer this care to the great number of potential patients is a challenge to be overcome in a way that is sustainable in the local context.

Finding ways to screen potential patients for diabetes and hypertension that does not overwhelm the local provider and support services is essential. Instead of using standard diagnostic testing as a first step, other groups have employed equation-based screening formulas supplemented after the first-pass with fasting blood sugar testing, blood pressure monitoring, and hemoglobin A1c testing of smaller subsets [9]. Whether our approach or a variation of the same as used in other projects is most appropriate depends in large part on the particulars of a given setting, the population being treated, local re- 
sources, as well as the availability of regional and national support.

A cornerstone of our approach was education using a peer-to-peer model that we believe had significant impact upon our patients and their surrounding communities, offering the promise of lasting benefit via deeper understanding and community-level insight. This is not a new concept overall as education has certainly been part of the treatment strategy in Latin America [10] as well as globally. Nevertheless, in combination with the resources and algorithm-guided care described here, the outcome measures speak to the impact of our approach.

The limitations in comparing the Dominican group of subjects to those in developed countries include that compliance in the latter relates to issues such as affordability of medications, availability of appropriate care providers, education and counseling to make patients aware of the benefits (short and long-term) of therapy, and what has been described as clinical inertia wherein proactive adjustment of therapy seems to lag behind what standard algorithms would predict [11]. In the Dominican cohort, medications were provided free of charge to subjects, very engaged and readily available providers were incorporated into the program from the outset, an educational program utilizing multimedia and simultaneously peer-to-peer learning delineated risks and benefits of therapy versus the lack thereof, and finally, the treatment algorithm played a central role in medical decision-making significantly diminishing clinical inertia.

Placing this within the context of the developing world, a varied and inhomogeneous grouping, a striking case is made here by way of proof of principle that facilitation of appropriate and adequate care as outlined above and which has been shown to work in the developed world produces similar results. A robust educational program is shown to provide a foundation of understanding of the diseases being addressed as well as the short and long-term benefits of doing so. Well-utilized local resources (i.e. health care workers and village-level clinical facilities) create a patient-provider dynamic that is necessary for dissemination as well as for sustaining this model. Defaulting to internationally agreed upon standards of care while allowing for individualization maintains the impetus to move treatment forward as indicated [12]. Finally, overcoming the significant hurdle of medication availability and affordability makes this intervenetion possible. This last point is a challenge to the sustainability of such a model. Also, developed world studies do point to diminishing treatment adherence with time which this cohort may also be subject to.

Numerous program challenges have included limited enrollment due to patient and physician transportation expense, initial laboratory unreliability that necessitated transporting blood samples 100 miles to a certified laboratory, general pessimism concerning patient medication adherence, the logistics of providing donated medication, periodic calibration of the ISTAT in the U.S. and the expense of strips used during the screening and follow-up visits ( $\$ 0.80$ - 1.00 per strip). The problem with blood test transportation has improved (lipid and liver function tests not withstanding) with the donation of a point of care A1c machine but the A1c cartridges still remain expensive at $\$ 6.50$ per cartridge. Punctuality and accurate record keeping is a work in progress although young intelligent physicians partially versed in Western medical care have been increasingly willing to comply with prescribed timelines and program goals.

The diabetes and hypertension protocols used initially, have been modified to reduce the number and frequency of follow-up visits with providers and to accelerate the titration of medications with the goal of maintaining patient safety while increasing staff capacity for further patient enrollment (Figures $\mathbf{2}$ and $\mathbf{3}$ ).

The second phase of the education process will utilize Care Maps, developed by Healthy Interactions and the American Diabetes Association. In this model small groups $(7-8)$ meet and a moderator (cooperadores de salud) facilitates the education session by reading questions and giving the group a chance to respond and discuss their answers.

Sustainability of the program is essential. Good relationships with local health authorities and education on infrastructure development plus continued program outcome success are mandatory. Sustainable health care initiatives require accessibility, high quality care, disruptive technology (cell phones, internet access, A1c and other blood test point of care testing), sustainable growth and affordability [13]. Our intent is to follow these principles for a sustainable solution and to eliminate U.S. personnel participation with time.

Ongoing monitoring of the program's outcomes will help inform its long term applicability to other settings. Essentials for another program include a well defined group of underserved patients, local political will, a native endocrinologist for consultation, a health provider network, local physician participation, medication and equipment donations, funding, and an organization able to provide support and coordination of services. The ILAC center has the advantage of its own network of health care providers but this can be replicated elsewhere. The cooperadores de salud are an integral and trusted part of the patients' communities - which is one of the main reasons for the program's success to date.

\section{CONCLUSION}

The application of a best practice approach, as has been 
shown to work in the developed world, has found traction in this poor rural setting in the Dominican Republic. That in itself is reason to be hopeful and to seek ways, and means, to take this forward. More patient enrollment and a closer study of cooperadores de salud-patient interaction is planned as well as enhancements of the data infrastructure. We would encourage replication of this program in other similar environments.

\section{ACKNOWLEDGEMENTS}

Many people deserve thanks you for their participation and program dedication. These include the cooperadores de salud Isabel Evangelista, Silvia De Los Angeles, Maria Matias, Maribel Garcia and Bernardino Cruz, our program nurse Ana Mercedes Munoz, the Ricketts family and Chicago Cubs Charities, Dr. Mercedes Capellan, Dr. Marcel Morel, Dr. Philip Frost and TEVA pharmaceuticals, Dr. Ken Culver and Mr. Jeff Spragens of The foundation for Peace, Mr. Radalme Pena, Ms. Pamela Schrier, Mr. David Mayo, Mrs. Frances Ann Filipi, Mr. Aaron Filipi, Dr. Viktor Reich, and Mrs. Jean Holtzen.

\section{REFERENCES}

[1] World Health Organization (2011) Diabetes. http://www.who.int/mediacentre/factsheets/fs312/en/

[2] Empirica (PowerPoint Slides) (2008) http://www.empirica.com.do/profesores/ffuentes/myfiles/pre sentaciones/DIABETES\%20-\%20NIP\%20PRESENTATI ON.pdf

[3] World Health Organization (2011) Fact Sheet No. 317.

[4] Acosta, D., Rottbeck, R., Rodriquez, J., Gonzalez, L.M. and Almanzar, M.R. (2010) The prevalence and social patterning of chronic diseases among older people in a population undergoing health transition. A 10/66 Group cross-sectional population-based survey in the Dominican Republic.

http://www.mendeley.com/research/the-prevalence-and-s ocial-patterning-of-chronic-diseases-among-older-people- in-a-population-undergoing-health-transition-a-1066-grou p-crosssectional-populationbased-survey-in-the-dominica $\underline{\text { n-republic/ }}$

[5] Personal Communication with Dr. Rodolfo Ortiz, Institute for Latin American Concern, Santiago, 9 or 10 November 2010 .

[6] National Bureau of Statistics of the Dominican Republic. The Rural Areas of the Dominican Republic. Statistical Overview. Year 2, No. 9. Monthly Bulletin. Dominican Republic. September 2008.

http://www.one.gov.do/index.php?module=articles\&func =view\&catid=207

[7] Cabellero, E., Ward, K., Ferro, A.M., Grant, R. and Koen, M. (2008) La horotoria de rosa. Joslin Diabetes Center, Boston.

[8] Guzman, J.R., Lyra, R., Aguilar-Salinas, C.A., Cavalcanti, S., Escano, F., Tambasia, M., et al. (2010) Treatment of type 2 diabetes in Latin America: A consensus statement by the medical associations of 17 Latin American countries. Revista Panamericana de Salud Pública, 28, 463471. doi:10.1590/S1020-49892010001200008

[9] Piette, J.D., Milton, E.C., Aiello, A.E., Mendoza-Avelares, M.O. and Herman, W.H. (2010) Comparison of three methods for diabetes screening in a rural clinic in Honduras. Revista Panamericana de Salud Pública, 28, 49-57. doi:10.1590/S1020-49892010000700008

[10] Garcia, R. and Suarez, R. (2007) Results of a Cuban education strategy in diabetes after 25 years of experience. Revista Cubana de Salud Pública, 33, April-June 2007.

[11] Miccoli, R., Penno, G. and Del Prato, S. (2011) Multidrug treatment of type 2 diabetes: A challenge for compliance. Diabetes Care, 34, S231-S235.

[12] Davies M. (2004) The reality of glycaemic control in insulin treated diabetes: Defining the clinical challenges. International Journal of Obesity and Related Metabolic Disorders, 28, S14-S22.

[13] Himalayan Cataract Project (2009) All about vision. http://www.allaboutvision.com/conditions/cataract-surger y-cost.htm 